\title{
Bacteraemia in patients admitted to an urban hospital in West Africa
}

\author{
Philip C Hill*1, Charles O Onyeama ${ }^{1}$, Usman NA Ikumapayi ${ }^{1}$, \\ Ousman Secka1, Samuel Ameyaw ${ }^{1}$, Naomi Simmonds ${ }^{2}$, Simon A Donkor, \\ Stephen R Howie ${ }^{1}$, Mary Tapgun ${ }^{1}$, Tumani Corrah $^{1}$ and Richard A Adegbola ${ }^{1}$
}

\author{
Address: ${ }^{1}$ Bacterial Diseases Programme, Medical Research Council Laboratories, Banjul, The Gambia and ${ }^{2}$ The Royal Free and University College \\ Medical School, London, UK \\ Email: Philip C Hill* - phill@mrc.gm; Charles O Onyeama - coonyeama@gmail.com; Usman NA Ikumapayi - uikumapayi@mrc.gm; \\ Ousman Secka - osecka@mrc.gm; Samuel Ameyaw - skahealthandlife@yahoo.com; Naomi Simmonds - naomi_simmonds@hotmail.com; \\ Simon A Donkor - sdonkor@mrc.gm; Stephen R Howie - showie@mrc.gm; Mary Tapgun - mtapgun@mrc.gm; \\ Tumani Corrah - tcorrah@mrc.gm; Richard A Adegbola - radegbola@mrc.gm \\ * Corresponding author
}

Published: 26 January 2007

BMC Infectious Diseases 2007, 7:2 doi:10.1 186/147/-2334-7-2

This article is available from: http://www.biomedcentral.com/I47I-2334/7/2

(c) 2007 Hill et al; licensee BioMed Central Ltd.

This is an Open Access article distributed under the terms of the Creative Commons Attribution License (http://creativecommons.org/licenses/by/2.0), which permits unrestricted use, distribution, and reproduction in any medium, provided the original work is properly cited.
Received: II August 2006

Accepted: 26 January 2007

\begin{abstract}
Background: Few studies on bacteraemia in Africa have been published. We aimed to prospectively identify the causative organisms of bacteraemia in The Gambia and their relation to clinical diagnoses, outcome and antimicrobial susceptibility.

Methods: Between November 2003 and February 2005 we studied those admitted to the Medical Research Council hospital who were suspected of having bacteraemia. We documented clinical features, outcome, pathogens identified and their susceptibility patterns, and searched for factors associated with bacteraemia.
\end{abstract}

Results: 871 patients were admitted and had a blood culture taken. The median age was 2 years (range 2 months to 80 years) and 36 of I I 9 tested were HIV positive; 54.5\% were male. 297 (34\%) had a positive result and 93 (10.7\% overall) were considered a genuine pathogen. Those with bacteraemia were more likely to die in hospital $(O R 2.79 ; 1.17-6.65, p=0.017)$ and to have a high white cell count (WCC; OR I.81;95\% Cl I.09-3.02; $\mathrm{p}=0.022$ ). Three organisms accounted for $73 \%$ of bacteraemias: Streptococcus pneumoniae $(45.2 \%)$, Staphylococcus aureus $(18.3 \%)$ and Escherichia coli (9.7\%) while non-typhoidal salmonellae (NTS) accounted for $8.6 \%$. Antimicrobial susceptibility of S. pneumoniae was very high to penicillin (97.5\%); high resistance was found to cotrimoxazole. S. aureus was generally highly susceptible to cloxacillin, gentamicin and chloramphenicol. E. coli and NTS were all susceptible to ciprofloxacin and mostly susceptible to gentamicin. Thirteen (33\%) S. pneumoniae isolates were of serotypes contained in a 7-valent pneumococcal conjugate vaccine and 20 (5I.3\%) were of the same serogroup.

Conclusion: In The Gambia, those with bacteraemia are more likely than those without to die in hospital and to have a raised peripheral blood WCC. S. pneumoniae is the most common organism isolated. Introduction of a pneumococcal conjugate vaccine can be expected to lead to a reduction in disease incidence. 


\section{Background}

There is a large excess of mortality in sub-Saharan Africa, particularly in children: under 5 year old mortality rates of $100-250$ per 1,000 compare with $10-30$ per 1,000 in developed countries [1]. It is increasingly clear that invasive bacterial infections are a major contributor to this excess [2], with incidence rates confirmed to be much higher than those reported in developed countries [3]. However there is a paucity of information as to the relative contribution of different organisms to bacterial infections in sub-Saharan Africa and how this varies across a full range of age groups.

The virtual elimination of Haemophilus influenzae type b (Hib) disease in The Gambia [4] has changed the relative importance of other pathogens in the etiology of invasive bacterial infection. It is thought that Streptococcus pneumoniae is probably the predominant organism, as indicated by the success of a 9-valent pneumococcal conjugate vaccine in children, which included an overall mortality benefit [5]. We prospectively studied patients admitted to the Medical Research Council (MRC) hospital in Greater Banjul (the opposite end of the country to the location of the pneumococcal vaccine trial) who were suspected of having bacteraemia, to identify the causative organisms in relation to clinical diagnoses and their antimicrobial susceptibility patterns, and to identify differences between those with bacteraemia and those without.

\section{Methods}

\section{Setting}

The Gambia is situated $12^{\circ}$ north of the equator and has a limited rainy season from June to October and most malaria transmission occurs from July to November. The MRC in The Gambia has a 42-bed ward which serves as a primary and tertiary health facility for a mainly urban population. It does not provide any surgical, obstetric or neonatal care. Such patients are referred to the Royal Victoria Teaching Hospital in Banjul, which has facilities to manage them.

\section{Participants}

We conducted a study to assess clinical and bacteriological characteristics of bacteraemia episodes in patients admitted at the MRC ward from November 2003 to February 2005. The decision to take blood samples for culture rested solely on the attending physician's clinical impression and judgment. Verbal consent was obtained prior to blood sampling. The study was approved by the joint Gambia government-MRC ethics committee.

\section{Data gathering}

Case notes of all patients admitted were reviewed daily to identify those who had blood cultures as part of their routine workup. We developed, piloted and finalised a stand- ardized questionnaire to record socio-demographic characteristics, clinical symptoms, tobacco and alcohol use, prior antibiotics, steroid and immunosuppressive agents use, history of concurrent medical conditions (e.g. diabetes, cancer, HIV, sickle cell disease), anthropometric and physical signs and the results of investigations. When antibiotics were prescribed, the name, route and time of administration were noted. The clinical diagnosis and inhospital outcome were recorded. An infectious diseases physician confirmed the final diagnosis for each case after review of the respective form. The decision to request any investigation was based solely on the attending physician's clinical judgment and each patient received routine medical care.

\section{Blood cultures}

Bacterial isolates were obtained from blood using an automated blood-culture system BACTEC 9050, (Becton Dickinson, Temse, Belgium). Commercially produced BD BACTEC $^{\mathrm{Tm}}$ PEDS PLUS ${ }^{\mathrm{TM}} / \mathrm{F}$ culture vials were used for specimens obtained from children aged one month to 15 years and BD BACTEC ${ }^{\text {TM }}$ Plus Aerobic/F* and Plus Anaerobic/F* culture vials for specimens from adults aged $>15$ years by direct inoculation of culture media at the patient's bedside following manufacturer's instructions for quality control and blood volume requirements. Whenever in sufficient blood volume was obtained only the Plus Aerobic/F* vial was inoculated. Whenever there was shortage of commercial vials each of Tryptone Soya Broth (TSB) and Brain Heart Infusion (BHI) culture media were used for specimens from adults and TSB only was used for specimens from children. We used standard microbiological procedures as described previously [6]. Further identification was by cultural morphology and biochemical methods. In addition, serotyping of the pneumococcal isolates was performed with capsular and factor-typing sera (Statens Serum Institut, Copenhagen, Denmark) using an antibody coated latex agglutination assay. Antimicrobial sensitivity patterns were determined by Kirby-Bauer disk diffusion test using interpretative criteria described previously [6]. The microbiology laboratory submits to the external quality assurance programme of the United Kingdom National External Quality Assessment Service.

Isolates were classified as contaminants if they were known to be a common skin contaminant, if there was scanty growth not on the line of inoculum that failed to grow on subculture, or if the isolate was obtained from the TSB aerobic bottle only after both bottles (TSB and BHI) had been inoculated with blood.

Malaria slides were stained by Field's stain and examined using a $\times 100$ oil immersion lens. Testing for HIV-1 or HIV-2 infection was by competitive enzyme linked immunosorbent assays (Wellcome Laboratories, Kent, UK) and 
Western blot (Diagnostics Pasteur, Marnes-la-Coquette, France).

\section{Data analysis}

All data were double-entered into an ACCESS database and checked for errors. We compared those with bacteraemia with those without. Weight for age was standardized using the CDC 2000 growth reference [7]. We defined the wet season as from June to November and the dry season from December to May. We defined tachycardia as $>=160$ Beats per minute in infants under 12 months of age, 140 in 1 to 5 year old children and 120 in those 6 years old and over. A raised respiratory rate was $>50$ breaths per minute in infants and $>40$ in other age groups. A clinical diagnosis of severe malnutrition was defined as either visible severe wasting or Kwashiokor. Septicaemia was defined as the presence of bacteraemia in the absence of a focus of infection. We assessed possible risk factors for bacteraemia by Chi-Square test and, in addition to age and sex that were included a priori, considered those with a p value of $<=0.15$ for a multi-variable logistic regression model. The explanatory variables in the final adjusted model were age (continuous variable), sex, temperature (categorical variable) and white cell count (categorical variable). A separate model compared the outcome of those with bacteraemia to those without, adjusting for the above variables. Analyses were conducted using Stata software (version 8; Stata Corp, College Station, TX).

\section{Results}

During the study period, 2712 patients were admitted to the MRC ward, the median age was 5 years (range 2 months to 80 years) and 871 underwent phlebotomy for blood culture. The median age of these patients was 2 years (range 2 months to 80 years) and $54.5 \%$ were male (table 1). Nine patients had taken corticosteroids in the last month, one had a history of malignancy, 6 were known to be diabetic. Two hundred and forty nine patients had a screening test for sickle cell disease, 58 $(23.3 \%)$ were positive. The median duration of stay was 4 days (range: 1 to 45 days). Forty nine (5.5\%) patients died in hospital. The overall mortality in the hospital was $6 \%$ during the period. One hundred and nineteen patients had known HIV status or were tested during the admission because of one or more clinical indication; 36 (30.2\%) were HIV positive.

Of those who had a blood culture taken, 297 (34\%) had a positive result and 93 (10.7\% overall) were considered to be a genuine pathogen (Figure 1). Those whose blood culture grew a contaminant were excluded from further analyses because of the possibility of masking of a true bacteraemia. Of those with bacteraemia, the same organism was obtained from another normally sterile site in 12 $(12.9 \%)$ cases.
Those with bacteraemia were more likely to die in hospital and, while this was not significant by Chi-Square, in an adjusted model it was significant (OR 2.74; 95\% CI 1.15$6.53 ; \mathrm{p}=0.022$ ). Those with bacteraemia were more likely to have a temperature $>=38^{\circ} \mathrm{C}$, although in the adjusted model this lost significance (OR 1.42; 95\% CI 0.9-2.25; p $=0.14$ ), having been subject to confounding by WCC. Those with a WCC of at least $10 \times 10^{9} / \mathrm{L}$ at presentation were also more likely to be bacteraemic, remaining significant in the adjusted model (OR 1.81;95\% CI 1.09-3.02; $\mathrm{p}=0.022$ ). For those with a WCC of at least $15 \times 10^{9} / \mathrm{L}$, the adjusted OR was 1.97 (95\% CI 1.23-3.15; $\mathrm{p}=0.005)$. Almost $40 \%$ of those in the study had a clinical diagnosis of pneumonia and the distribution of clinical diagnoses were similar between the two groups. Of those with pneumonia $276(83 \%)$ had an xray, and $263(95.3 \%)$ were assessed as being abnormal.

Three organisms combined to account for $73 \%$ of all bacteraemias (table 2); S. pneumoniae (45.2\%), Staphylococcus aureus (18.3\%) and Escherichia coli $(9.7 \%)$ while nontyphoidal salmonellae(NTS) accounted for $8.6 \%$ of isolates. Of note, $\mathrm{H}$. influenzae accounted for 2 isolates ( $1 \mathrm{Hib}$ and $1 \mathrm{Hi}$ non-serotypable) and Neisseria spp. accounted for 1 isolate (Figure 1). None of the organisms detected were more likely to be identified in the wet season. In those who were bacteraemic, 2 of 10 with malaria on blood film had NTS as opposed to 4 of 70 who had a negative blood film, although this difference was not significant $(\mathrm{p}=0.12)$. S. pneumoniae and $S$. aureus were relatively evenly distributed across the age groups. In contrast, 5 of the 9 E. coli isolates were found in infants, although only one was under 6 months of age. Of the $8(8.6 \%)$ bacteraemic patients who died, 3 had $S$. pneumoniae, 2 had $S$. aureus, 2 had NTS and 1 had Proteus spp.

Of 39 isolates of S. pneumoniae that were characterised further by serotyping, 14 serotypes/groups $(1,5,14,23 \mathrm{~F}, 6 \mathrm{~A}, 19 \mathrm{~A}, 6 \mathrm{~B}, 4,9 \mathrm{~V}, 9 \mathrm{~L}, 10,15,18 \mathrm{C}, 22)$ were identified, two (1 and 5) accounted for $16(41 \%)$ of the isolates. Thirteen (33\%) were of serotypes contained in a 7 -valent pneumococcal conjugate vaccine $(4,6 \mathrm{~B}, 9 \mathrm{~V}, 14$, $18 \mathrm{C}, 19 \mathrm{~F}$, and $23 \mathrm{~F})$ and $20(51.3 \%)$ were of a 7 -valent vaccine serogroup.

Isolates of S. pneumoniae (39, 97.5\%) were highly susceptible to penicillin (Table 3 ), ampicillin $(100 \%)$ and chloramphenicol (97.6\%). They were moderately susceptible to tetracycline $(65.9 \%)$ and only poorly susceptible to cotrimoxazole (5\%). S. aureus isolates were all susceptible to cloxacillin, gentamicin and chloramphenicol; moderately susceptible to co-trimoxazole (66.7\%) and poorly susceptible to tetracycline $(33 \%)$ and penicillin $(8 \%)$. Among Gram-negative bacteria, all isolates of E. coli (9) and NTS $(5-7 \%)$ were susceptible to ciprofloxacin. In addition, $E$. 
Table I: Characteristics of the patients admitted with suspected bacteraemia.

\begin{tabular}{|c|c|c|c|c|}
\hline Characteristics & All $(n=87 I)$ & Bacteraemia $(n=93)$ & non-Bacteraemia $(n=574)$ & p value \\
\hline \multicolumn{5}{|l|}{ Age } \\
\hline $2 \mathrm{mo}-11 \mathrm{mo}$ & $200(23.0)$ & $24(25.8)$ & $110(19.2)$ & \\
\hline II mo-23 mo & $207(23.8)$ & $18(19.4)$ & $127(22.1)$ & \\
\hline $2-5$ years & $166(19.1)$ & $23(24.7)$ & $112(19.5)$ & \\
\hline $6-15$ years & $113(13.0)$ & $13(14.0)$ & $77(13.4)$ & \\
\hline$>15$ years & $185(21.2)$ & $15(16.1)$ & $148(25.8)$ & 0.185 \\
\hline Male & $475(54.5)$ & $59(63.4)$ & $312(54.4)$ & 0.102 \\
\hline Wet season admission & $533(61.2)$ & $60(64.5)$ & $405(70.6)$ & 0.295 \\
\hline Duration of symptoms $<7$ days $(n=849)$ & $459(54.1)$ & $43(47.8)$ & $307(55.1)$ & 0.252 \\
\hline Weight for age $z$ score $<-2(n=693)$ & $373(53.8)$ & $39(51.3)$ & $221(51.0)$ & 0.87 \\
\hline Temperature $>=38^{\circ} \mathrm{C}(n=863)$ & $364(42.2)$ & $48(51.6)$ & $229(40.3)$ & 0.041 \\
\hline Tachycardia $(n=738)$ & $253(34.2)$ & $38(46.9)$ & $182(37.3)$ & 0.141 \\
\hline Raised respiratory rate $(n=677)$ & $347(51.3)$ & $51(62.2)$ & $247(55.2)$ & 0.297 \\
\hline \multicolumn{5}{|l|}{ White Blood Cell Count $(n=852)$} \\
\hline$>=10 \times 109 / \mathrm{L}$ & $484(56.8)$ & $64(70.3)$ & $306(54.6)$ & 0.005 \\
\hline$>=15 \times 109 / \mathrm{L}$ & $290(34.0)$ & $45(49.5)$ & $173(30.8)$ & $<0.0001$ \\
\hline Haemoglobin < 8 g/L $(n=602)$ & $192(32.0)$ & $30(41.1)$ & $|2|(31.8)$ & 0.121 \\
\hline Sickle cell test positive $(n=243)^{a}$ & $57(6.5)$ & $8(8.6)$ & $13(2.2)$ & 0.144 \\
\hline Died in hospital & $49(5.5)$ & $8(8.3)$ & $25(4.3)$ & 0.081 \\
\hline \multicolumn{5}{|l|}{ Specific Clinical Diagnoses } \\
\hline Pneumonia & $332(38.1)$ & $40(43.0)$ & $207(36.1)$ & 0.198 \\
\hline Malaria $(n=676 \text { tested })^{a}$ & $101(11.6)$ & $10(10.8)$ & $65(11.3)$ & 0.82 \\
\hline Severe Malnutrition & $68(7.8)$ & $8(8.6)$ & $50(8.7)$ & 0.975 \\
\hline Tuberculosis & $34(3.9)$ & $\mathrm{I}(\mathrm{I} . \mathrm{I})$ & $29(5.1)$ & 0.086 \\
\hline HIVIAIDS $(\mathrm{n}=119 \text { tested })^{\mathrm{a}}$ & $36(4.1)$ & $6(6.4)$ & $25(4.4)$ & 0.397 \\
\hline Gastroenteritis & $36(4.1)$ & $\mathrm{I}(\mathrm{I} .1)$ & $24(4.2)$ & 0.145 \\
\hline Urinary tract infection & $25(2.9)$ & $2(2.2)$ & $19(3.3)$ & 0.573 \\
\hline Nephrotic/Nephritic syndrome & $23(2.6)$ & $\mathrm{I}(\mathrm{I} . \mathrm{I})$ & $17(3.0)$ & 0.297 \\
\hline Skin/soft tissue infection & $20(2.3)$ & $3(3.2)$ & $15(2.6)$ & 0.74 \\
\hline Meningitis & $13(1.5)$ & $\mathrm{I}(\mathrm{I} . \mathrm{I})$ & $9(1.6)$ & 0.716 \\
\hline Upper respiratory tract infection & $12(1.4)$ & 0 & $9(1.6)$ & 0.252 \\
\hline Hepatititis & $12(1.4)$ & 0 & $10(1.7)$ & 0.235 \\
\hline Bone/Joint infection & $9(1.0)$ & 0 & $6(1.0)$ & 0.387 \\
\hline
\end{tabular}

a For sickle cell, malaria and HIV tests, because the investigations were driven by clinical suspicion, the denominator for the $\%$ calculations remained the total number in each category.

coli isolates were highly susceptible to gentamicin $(88.9 \%)$ and moderately susceptible to chloramphenicol (66.7\%), poorly susceptible to co-trimoxazole $(22 \%)$ and completely non-susceptible to ampicillin. Non-typhoidal isolates were also susceptible to gentamicin (100\%), tetracycline $(80 \%)$, co-trimoxazole $(71 \%)$, chloramphenicol (66.7\%) and ampicillin (57\%).

\section{Discussion}

In this study we have shown that Gambian patients with bacteraemia are more likely than those without to die in hospital and to have a raised peripheral blood WCC. Three organisms accounted for $73 \%$ of all bacteraemias; $S$. pneumoniae accounted for $45 \%$. This hospital based study emphasizes the dominance of $S$. pneumoniae in the etiology of bacteraemia in The Gambia, consistent with previous etiology studies in this country [8-10].
S. pneumoniae has been identified as the dominant isolate in bacteraemia elsewhere in Africa. Berkowitz [11] identified bacteraemia in 315 (5.8\%) of 5397 children admitted to a South African hospital; $23 \%$ died. S. pneumoniae accounted for $23 \%$ of isolates, S. aureus $6 \%$, Salmonella species $20 \%$, E. coli 13\%, and H. influenzae $14 \%$. Cotton et al [12] documented 132 episodes of communityacquired bacteraemia in hospitalized South African children; $12 \%$ died. S. pneumoniae accounted for $33 \%$ of pathogens isolated, S. aureus $14 \%, N$. meningitidis $11 \%$ and other Gram negative organisms $18 \%$. Berkley et al [3] found that $866(5.9 \%)$ of 14,787 children over 60 days of age admitted to a rural hospital in Kenya had bacteraemia. S. pneumoniae accounted for $30 \%$ of isolates, S. aureus $7 \%$, other Gram positive cocci 6\%, NTS 19\%, H. influenzae $15 \%$, and E. coli $10 \%$. In Malawi, Archibald et al [13] found blood stream infection in $70(30 \%)$ of 233 febrile 


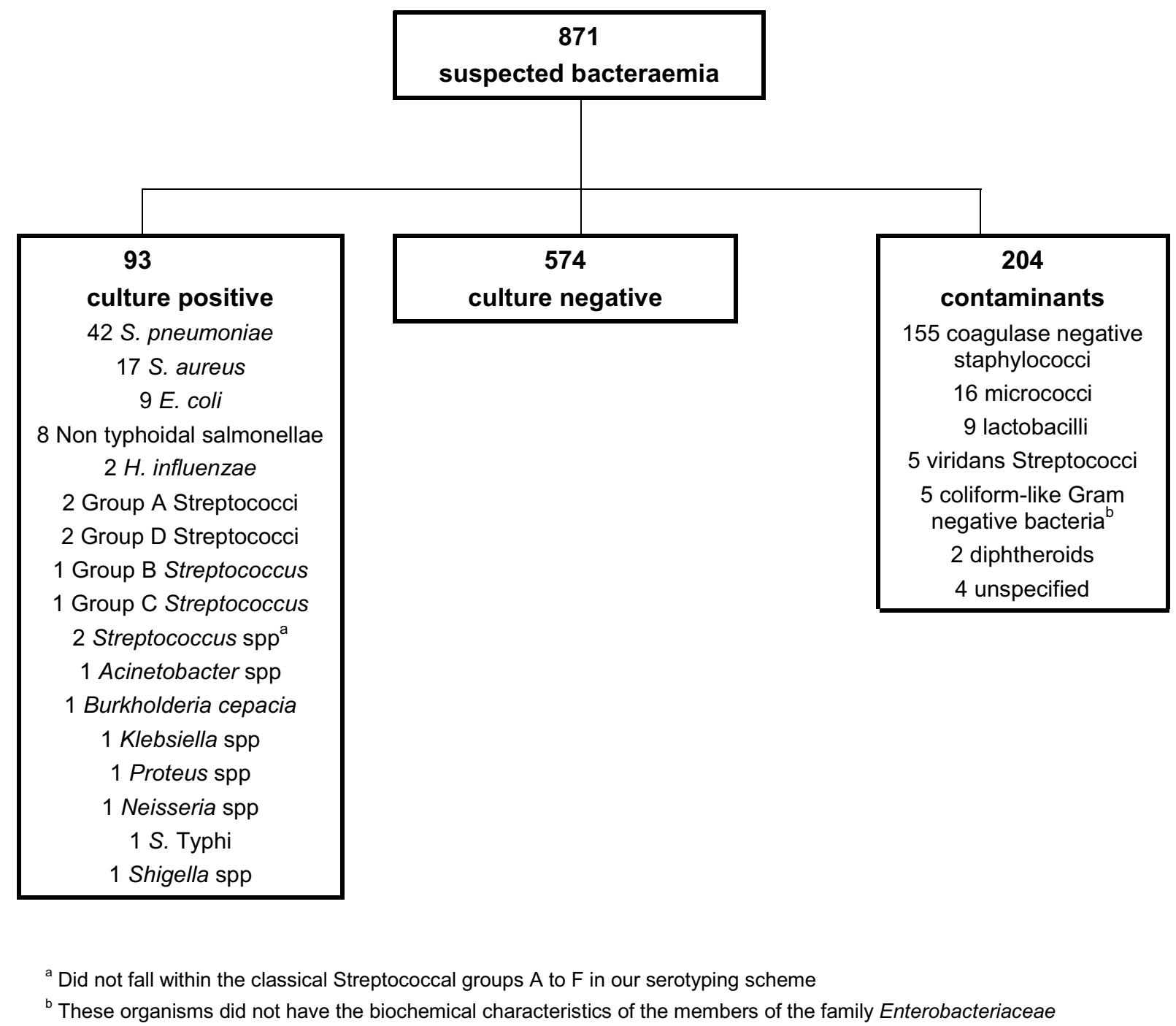

Figure I

Flow diagram of suspected bacteraemia patients according to blood culture results.

adults admitted to hospital, 70\% of whom were HIV positive. S. pneumoniae accounted for 33\% of isolates, Salmonella species $19 \%$ and Gram positive cocci only $4 \%$. M. tuberculosis accounted for $29 \%$ of isolates, found exclusively in HIV positive patients.

Salmonella species have predominated in several other African studies, particularly in high HIV prevalence settings. Bahwere et al [14] identified 126 (15.9\%) bacteraemias in 779 children admitted to a hospital in Congo; $19.4 \%$ died. Salmonella species accounted for $44 \%$ of isolates. Gram positive bacteria accounted for only $10 \%$ of isolates. Ghiorghis et al [15] identified bacteraemia in $49(7.7 \%)$ of 634 febrile children at an Ethiopian hospital. Of the organisms isolated, Salmonella species accounted for $57 \%$, streptococci $16 \%$, staphylococci $14 \%$, and $E$. coli $6 \%$ and other Gram negative species $6 \%$. Walsh et al [16] reported that of 365 positive isolated from children in Malawi, NTS accounted for $38 \%$ of isolates, $H$. influenzae 6\%, other Gram negative species 29\%, S. pneumoniae $16 \%$, other streptococci $8 \%$ and S. aureus $2 \%$. Gordon et al [17] identified 449 pathogens from the blood of adult patients in Blantyre: NTS accounted for $37 \%$ of isolates and 30\% were S. pneumoniae. NTS accounted for 70$80 \%$ of all paediatric blood culture isolates in a hospital in Western Zaire [18]. S. aureus was identified by Meremikwu et al [19] as the cause of bacteraemia in $48.7 \%$ of bacteraemias in children admitted to a Nigerian hospital, $44 \%$ were neonates. Differing criteria for patient selection, variation in the standardisation of methods and bacterio- 
Table 2: Number of isolates/groups of isolates and their relative proportion (\%) in each of 5 age bands and in each of 6 clinical diagnostic categories for those with Bacteraemia $(n=93)$.

\begin{tabular}{|c|c|c|c|c|c|c|}
\hline & \multicolumn{6}{|c|}{ Organism/group, n (\% of row) } \\
\hline & S. pneumoniae & S. aureus & Other Gram +ve & E. coli & NTa salmonellae & Other Gram -ve \\
\hline \multicolumn{7}{|l|}{ Age group } \\
\hline $2 \mathrm{mo}-11 \mathrm{mo}$ & $9(37.5)$ & $2(8.3)$ & $4(16.7)$ & $5(20.8)$ & $2(8.3)$ & $2(8.3)$ \\
\hline $11 \mathrm{mo}-23 \mathrm{mo}$ & $8(44.4)$ & $4(22.2)$ & I (5.6) & I (5.6) & $2(11.1)$ & $2(11.1)$ \\
\hline $2-5$ years & $12(52.2)$ & $4(17.4)$ & I (4.4) & $3(13.0)$ & I (4.4) & $2(8.7)$ \\
\hline $6-15$ years & $7(53.4)$ & $3(23.1)$ & I (7.7) & 0 & 0 & $2(15.4)$ \\
\hline$>15$ years & $6(40.0)$ & $4(26.7)$ & I (6.7) & 0 & $3(20.0)$ & I (6.7) \\
\hline All & $42(45.2)$ & $17(18.3)$ & $8(8.6)$ & $9(9.7)$ & $8(8.6)$ & $9(9.7)$ \\
\hline \multicolumn{7}{|l|}{ Diagnostic category } \\
\hline Pneumonia & $30(78.9)$ & $4(10.5)$ & $2(5.3)$ & 0 & 0 & $2(5.3)$ \\
\hline Sepsis & $7(29.2)$ & $6(25.0)$ & $3(12.5)$ & $2(8.3)$ & $3(12.5)$ & $3(12.5)$ \\
\hline Malaria & 0 & I (14.3) & 0 & $2(28.6)$ & $2(28.6)$ & $2(28.6)$ \\
\hline HIVIAIDS & I (I6.7) & $2(33.3)$ & 0 & I (I6.7) & $2(33.3)$ & 0 \\
\hline Severe Malnutrition & $2(40.0)$ & I (20.0) & $2(40.0)$ & 0 & 0 & 0 \\
\hline Other & $2(15.4)$ & $3(23.1)$ & I (7.7) & $4(30.8)$ & I (7.7) & $2(15.4)$ \\
\hline
\end{tabular}

a Non-typhoidal

logical techniques used may explain some of the differences seen between studies.

Others have identified risk factors for bacteraemia in African settings. Ghiorghis et al [15] found no difference in nutritional status, age or temperature between febrile bacteraemic cases and controls, but did find increased WCC in those with salmonellae and in those with E. coli. Bahwere et al [14], in their study dominated by Enterobacteraciae, also found no association with severe malnutrition. However, Cotton et al [12], Berkowitz et al [11] and Friedland [20] identified severe malnutrition as a risk factor for bacteraemia and death in South Africa. Archibald et al [13] identified HIV positivity as a risk factor for bacteraemia in Malawi. HIV and malnutrition have been shown to be independent risk factors for bacteraemia by Berkley et al [3].

In contrast to our study, seasonal variation in the etiology of bacteraemia has been demonstrated in sub-Saharan Africa. Bell et al [21], in a Malawi population with high rates of HIV positivity, found that NTSpredominated in the wet season while $S$. pneumoniae predominated in the dry season. High prevalence of NTS in the wet season in sub-Saharan Africa is thought to be associated with malaria [22-24], although other, often site-specific, factors should be considered as well. We had too few cases of NTS to assess seasonal variation properly in this study.

Other studies from The Gambia confirm that high resistance to penicillin is not yet prevalent here $[10,6]$. In contrast, high resistance was found to co-trimoxazole, which is widely used at primary health care level. Evaluation of an alternative antimicrobial for community management of non-severe bacterial infections is now underway. Although numbers of isolates were small, $S$ aureus was in general highly susceptible to cloxacillin, gentamicin and chloramphenicol. Penicillin was the least effective antibiotic. Unlike several other developing countries where resistance has been reported to these antimicrobials, cheap and widely available antibiotics remain effective in The Gambia. A larger study to give more precise estimates

Table 3: Antibiotic susceptibility of the 3 major organisms isolated and non-typhoidal salmonellae in relation to the number tested.

\begin{tabular}{lcccccccc}
\hline Organism & \multirow{2}{*}{ Penicillin } & Ampicillin & Cloxacillin & Cotrim & Gent & Chloram & Tetra & Cipro \\
\hline S. pneumoniae & $39 / 40$ & $42 / 42$ & ND & $2 / 39$ & ND & $40 / 41$ & $27 / 41$ & ND \\
S. aureus & $4 / 14$ & ND & I4/14 & $10 / 15$ & $15 / 15$ & $15 / 15$ & $5 / 15$ & ND \\
E. coli & ND & $0 / 9$ & ND & $2 / 9$ & $8 / 9$ & $6 / 9$ & ND & $9 / 9$ \\
NTa- & ND & $4 / 7$ & ND & $5 / 7$ & $7 / 7$ & $4 / 6$ & $4 / 5$ & $7 / 7$ \\
salmonellae & & & & & & &
\end{tabular}

\footnotetext{
a Non-typhoidal
} 
of antimicrobial susceptibility of isolates of E. coli and NTS is required.

While this study represents 'real life' clinical practice in this hospital and our data were prospectively gathered, our approach has several limitations. A more formal study would set strict criteria for taking blood for culture and standardize all clinical and laboratory procedures. Such an approach would enable certain tests to be routine, such as HIV testing, and may involve sending certain isolates to a reference laboratory for further identification. It is estimated that $5-10 \%$ of inpatients in our hospital are HIV positive (unpublished data). Despite having a standardized form, we did not ask the clinician to record his/her primary reason for taking a blood culture (we felt this would be difficult to categorize), although $47 \%$ of patients had a temperature of $37.5^{\circ} \mathrm{C}$ or more and $13 \%$ of these had a pathogen isolated. As $80 \%$ of our study subjects were under 5 years of age, interpretation of our findings in the older age groups should be done cautiously. We also cannot calculate an incidence rate of communityacquired bacteraemia as the source population of this hospital cannot be clearly defined and we did not obtain blood cultures from a representative sample of outpatient attendees [25].

\section{Conclusion}

This study confirms that bacteraemia is an important illness in hospital patients in The Gambia. It appears that the most effective way to combat common severe bacterial infections in Africa is through vaccination [26], a premise supported by the virtual elimination of Hib disease from The Gambia [4]. The success of the 9-valent pneumococcal conjugate vaccine against pneumococcal disease in The Gambia, [5] together with the results of the present study, dictate that the introduction of a pneumococcal vaccine into the routine vaccination schedule is a high priority. It is intended that the currently available 7 -valent pneumococcal conjugate vaccine will be introduced into The Gambia. This vaccine could be expected to protect against a substantial proportion of invasive pneumococcal disease and may lead to improvements in resistance to some antibiotics. However, it will not prevent disease by pneumococci of serotypes 1 and 5, the most prevalent serotypes in invasive pneumococcal disease in The Gambia [6]. Therefore we will monitor the introduction of this vaccine with a surveillance system to identify trends in the incidence of invasive pneumococcal disease and changes in serotype prevalence [27].

\section{Competing interests}

The author(s) declare that they have no competing interests.

\section{Authors' contributions}

PH conceived the study and was involved in the design, clinical aspects, data analysis and wrote the paper with $\mathrm{CO}$ and RA, who were responsible for the clinical aspects and laboratory aspects of the study respectfully. SA participated in the clinical aspects of the study. OS coordinated the lab work with UI and RA. NS developed the form for the study and piloted and finalized it. SD managed the data entry and verification and quality. SH was involved in the design of the study, in particular the study form, and was involved in the clinical care of the children. MT supervised the clinical aspects of the study with TC. All authors read and approved the final manuscript.

\section{Acknowledgements}

We thank Dr Robin Bailey for useful comments in the design of the form for this study. We thank the nursing staff on the MRC ward for accommodating the study. This study, and all the authors, were funded by the Medical Research Council (UK). The funder had no role in the study design, data collection, interpretation, writing of the manuscript or the decision to submit the study for publication.

\section{References}

I. World Health Organisation: World Health report 1998, Life in the 2 I st Century a vision for all. [http://www.who.int/whr//998/ en/index.html]. Cited 27.1 I.05

2. Mulholland EK, Adegbola RA: Bacterial infections-a major cause of death among children in Africa. N Eng J Med 2005, 352:75-77.

3. Berkley JA, Lowe BS, Mwangi I, Williams T, Bauni E, Mwarumba S, Ngetsa C, Slack MPE, Njenga S, Hart CA, Maitland D, English M, Marsh K, Scott JAG: Bacteremia among children admitted to a rural hospital in Kenya. N Eng J Med 2005, 352:39-47.

4. Adegbola RA, Secka O, Lahai G, Lloyd-Evans N, Njie A, Usen S, Oluwalana C, Obaro S, Weber M, Corrah T, Mulholland K, McAdam K, Greenwood B, Milligan PJ: Elimination of Haemophilus influenzae type b (Hib) disease from The Gambia after the introduction of routine immunization with a Hib conjugate vaccine: a prospective study. Lancet 2005, 366: |44- I50.

5. Cutts FT, Zaman SMA, Enwere G, Jaffar S, Levine OS, Okoko JB, Oluwanlana C, Vaughan A, Obaro S, Leach A, McAdam KP, Biney E, Saaka M, Onwuchekwa U, Yallop F, Pierce NF, Greenwood BM, Adegbola RA: Efficacy of nine-valent pneumococcal conjugate vaccine against pneumonia and invasive pneumococcal disease in The Gambia: randomized, double-blind, placebo-controlled trial. Lancet 2005, 365: I |39- I |46.

6. Adegbola RA, Hill PC, Secka O, Otu J, Sarr R, Sillah J, Lienhardt C, Corrah T, Manneh K, Drobniewski F, McAdam KP: Serotype and antimicrobial susceptibility patterns of isolates of Streptococcus pneumoniae causing invasive disease in The Gambia 1 996-2003. Trop Med Int H 2006, I I: I I 28-I I 35.

7. Vidmar S, Carlin J, Hesketh K, Cole T: Standardising anthropometric measures in children and adolescents with new functions for egen. Stata J 2004, 4:50-5.

8. Wall RA, Corrah PT, Mabey DCW, Greenwood BM: The etiology of lobar pneumonia in the Gambia. Bull WHO I 986, 64:553-558.

9. O'Dempsey TJ, McArdle TF, Lloyd-Evans N, Baldeh I, Lawrence BE, Secka O, Greenwood B: Pneumococcal disease among children in a rural area of West Africa. Pediatr Infect Dis J 1996, I 5:43 |-437.

10. Enwere G, Biney E, Cheung YB, Zaman SM, Okoko B, Oluwalana C, Vaughan A, Greenwood B, Adegbola R, Cutts FT: Epidemiologic and clinical characteristics of community-aquired invasive bacterial infections in children aged 2-29 months in The Gambia. Pediatr Infect Dis J 2006, 25:700-705.

II. Berkowitz FE: Bacteremia in hospitalized black South African children. Am J Dis Child 1984, I38:55 I-555.

12. Cotton MF, Burger PJ, Bodenstein WJ: Bacteraemia in children in the south-western Cape. A hospital-based survey. S Afr MedJ 1992, 8 I (2):87-90. 
13. Archibald LK, McDonald LC, Nwanyanwu O, Kazembe P, Dobbie H, Tokars J, Reller LB, Jarvis WR: A hospital-based prevalence survey of bloodstream infections in febrile patients in Malawi: implications for diagnosis and therapy. J Infect Dis 2000, I8I:|14|4-|420.

14. Bahwere P, Levy J, Hennart P, Donnen P, Lomoyo W, Dramaix-Wilmet M, Hemelof W, Butzler J, De Mol P: Community-acquired bacteremia among hospitalized children to rural central Africa. Int J Infect Dis 200।, 5: I80-188.

15. Ghiorghis B, Geyid A, Haile M: Bacteraemia in febrile outpatient children. East Afr Med J 1992, 69(2):74-77.

16. Walsh AL, Phiri AJ, Graham SM, Molyneux, Molyneux ME: Bacteremia in febrile Malawian children: clinical and microbiological features. Pediatr Infect Dis J 2000, 19:312-318.

17. Gordon MA, Walsh AL, Chaponda M, Soko D, Mbvwinji M, Molyneux ME, Gordon SB: Bacteraemia and mortality among adult medical admissions in Malawi-predominance of non-typhi salmonellae and Streptococcus pneumoniae. J Infect 200I, 42:44-49.

18. Green SDR, Cheesbrough JS: Salmonella bacteraemia among young children at a rural hospital in western Zaire. Ann Trop Paediatr 1993, 13:45-54.

19. Meremikwu MM, Nwachukwu CE, Asuquo AE, Okebe JU, Utsalo SJ: Bacterial isolates from blood cultures of children with suspected septicaemia in Calabar, Nigeria. BMC Infect Dis 2005, 5:II0-II4.

20. Friedland IR: Bacteraemia in severely malnourished children. Ann Trop Paediatr 1992, I 2:433-440.

21. Bell M, Archibald LK, Nwanyanwu O, Dobbie H, Tokars J, Kazembe PN, Reller LB, Jarvis WR: Seasonal variation in the etiology of bloodstream infections in a febrile inpatient population in a developing country. Int I infect Dis 200I, 5:63-69.

22. Mabey DCW, Brown A, Greenwood BM: Plasmodium falciparum malaria and Salmonella infections in Gambian children. J Infect Dis 1987, 155:|319-|321.

23. Lepage P, Bogaerts J, Van Goethem C, Ntahorutaba M, Nsengumuremyi F, Hitimana DG, Vandepitte J, Butzler JP, Levy J: Communityacquired bacteraemia in African children. Lancet 1987 I:| $|458-| 46 \mid$

24. Duggan MB, Beyer L: Enteric fever in young Yoruba children. Arch Dis Child 1975, 50:67-7I.

25. Brent AJ, Ahmed I, Ndiritu M, Lewa P, Ngetsa C, Lowe B, Bauni e, English M, Berkley JA, Scott JAG: Incidence of clinically significant bacteraemia in children who present to hospital in Kenya: a community-based observational study. Lancet 2006, 367:482-488.

26. Peltola $\mathrm{H}$ : Burden of meningitis and other severe bacterial infections of children in Africa: implications for prevention. Clin Infect Dis 200I, 31:64-75.

27. Steenhoff AP, Shah SS, Ratner AJ, Patil SM, McGowan KL: Emergence of vaccine-related pneumococcal serotypes as a cause of bacteraemia. Clin Infect Dis 2006, 42:907-9I4.

\section{Pre-publication history}

The pre-publication history for this paper can be accessed here:

http://www.biomedcentral.com/1471-2334/7/2/prepub

\section{Publish with Bio Med Central and every scientist can read your work free of charge}

"BioMed Central will be the most significant development for disseminating the results of biomedical research in our lifetime. "

Sir Paul Nurse, Cancer Research UK

Your research papers will be:

- available free of charge to the entire biomedical community

- peer reviewed and published immediately upon acceptance

- cited in PubMed and archived on PubMed Central

- yours - you keep the copyright
BioMedcentral 